\title{
Editorial
}

\section{Distributed information system}

\author{
Niv AHITUV \\ Tel-Aviv University, Tel-Aviv, Israel \\ Claremont Graduate School, Claremont, CA 91711, USA
}

Until the late 70's information system technology (i.e., computers, communications network, etc.) had not been flexible enough to adjust itself to various managerial styles, in particular to decentralized organizations. Computers were large and centralized; consequently, data bases and computing power could not be delegated to the end user. The rapid progress in the development of microcomputers and communications has brought technology to a state where management can subdue the hardware and software configuration to comply to its managerial approach. Information systems which are less centralized than in the past are labelled Distributed Information Systems (DIS).

A DIS is not necessarily a fully distributed system. It is an information system where the responsibility over some of its components has been delegated to end users. For instance, the requirement definition is performed by the user; the analysis, design, and programming are carried out by a central body; the routine operation is undertaken by the user; the insert of software changes and the upgrading of equipment is centrally managed. Such combinations of distributed and centralized activities are widely common. The major problems they raise are not technical but managerial - how can management decide what activities are to be distributed, and how distributed activities can still be controlled.

It can be observed that in parallel to the technological progress, the literature on DIS has passed through three generations. The first generation (the beginning of the 70's) focused on solving technical problems, e.g., how to connect computers

North-Holland

Human Systems Management 5 (1985) 275-276 and terminals deployed in distinct geographical locations.

The second generation (the late 70's) dealt with efficiency and economic problems, e.g., optimizing the distribution of computing facilities.

The third generation (the beginning of the 80's) directs its attention to managerial problems, i.e., how to devise a policy for DIS. Two notable papers in this area were written by Buchanan and Linowes [1]. They were the first to emphasize that an information system is not a rigid entity but a compound of a large number of activities; it entails that while a certain activity is distributed, another one can be centralized. The immediate conclusion is that questions of distribution policy, managing, and control are not less involved problems.

This issue of HSM focuses on DIS by publishing three papers dealing with various aspects of DIS. In a way, the three papers follow the 'third generation', namely, they concentrate on managerial problems rather than on technical ones. However, they are more specific than previous literature since they do not deal with distribution as an abstract concept but with segments of technology where distribution widely prevails. These are microcomputers and CAD/CAM (Computer Aided Design/Computer Aided Manufacturing).

The proliferation of microcomputers is believed to be the most influencial factor with regard to the growing use of DIS. In many cases, microcomputers are selected individually by an interested user. The paper by Arbel and Seidmann ('Designing a multi-micro distributed information system') provides a comprehensive approach to the design and selection of microcomputers. The approach is based on multicriteria decision making.

Borovits' paper ('Managing the distribution and use of microcomputers in the organization') copes with a very severe problem of uncontrolled proliferation of microcomputers. A survey done once in a university campus I am familiar with has 
observed that within a year 15 word-processors were purchased from 12 different vendors. Such phenomenon is not very rare. Borovits' paper suggests a framework through which management can control the acquisition and deployment of microcomputers. A major point in this framework is the establishing of an information center - an organizational unit that supports end users.

The third paper (Ahituv and Ronen, 'Centralization and distribution of CAD/CAM systems') centers on a problem of allocating CAD/CAM facilities. These can be fully integrated, partly integrated or totally separate; decisions on CAD/ CAM acquisition can be centralized or delegated to users. The similarity between problems encountered in devising a CAD/CAM policy and a
DIS policy is very high. The paper, therefore, starts by 'converting' the Buchanan and Linowes' approach to a CAD/CAM environment. It then adds some points which are unique to CAD/CAM.

The three papers are very relevant to the main motive of HSM - management of high-tech. I hope they will contribute to the advancement of this issue.

\section{References}

[1] Buchanan, J.R. and R.G. Linowes, Understanding distributed data processing, Haryard Business Review, July-Aug. (1980) 143-153 and Making distributed data processing work, Harvard Business Review, Sept.-Oct. (1980) 143-161. 